\title{
Stability of an alternative functional equation related to Jensen's functional equation
}

\author{
Choodech Srisawat \\ Department of Mathematics, Faculty of Science, Udon Thani Rajabhat University, \\ Udon Thani 41000 Thailand \\ e-mail: ch.srisawat@gmail.com
}

Received 29 Jul 2017

Accepted 30 Sep 2017

ABSTRACT: Given an integer $\lambda \neq 1$, we verify the Hyers-Ulam stability of the alternative Jensen's functional equations $f\left(x y^{-1}\right)-2 f(x)+\lambda f(x y)=0$ where $f$ is a mapping from a 2-divisible group to a Banach space and $\lambda$ is an integer.

KEYWORDS: alternative equation, Jensen's functional equation

MSC2010: 39B82 39B72

\section{INTRODUCTION}

The alternative Cauchy functional equations have been widely studied. For instance, Kannappan and Kuczma ${ }^{1}$ studied the solutions of the alternative Cauchy functional equations of the form

$$
\begin{aligned}
(f(x+y)-a f(x)-b f(y)) & \\
& (f(x+y)-f(x)-f(y))=0,
\end{aligned}
$$

where $f$ is a function from an abelian group to a commutative integral domain and of characteristic zero. Ger ${ }^{2}$ extended (1) to the alternative functional equation

$$
\begin{aligned}
& (f(x+y)-a f(x)-b f(y)) \\
& \quad(f(x+y)-c f(x)-\mathrm{d} f(y))=0 .
\end{aligned}
$$

Forti $^{3}$ then established the general solution of the alternative Cauchy functional equations

$$
\begin{aligned}
(c f(x+y)-a f & (x)-b f(y)-d) \\
& (f(x+y)-f(x)-f(y))=0 .
\end{aligned}
$$

Nakmahachalasint ${ }^{4}$ first studied the solutions of an alternative Jensen's functional equations of the form

$$
f(x) \pm 2 f(x y)+f\left(x y^{2}\right)=0
$$

on a semigroup which extended the work in Refs. 5, 6 on the classical Jensen's functional equation

$$
f\left(x y^{-1}\right)-2 f(x)+f(x y)=0
$$

on a group. Nakmahachalasint ${ }^{7}$ also investigated the Hyers-Ulam stability of the alternative Jensen's functional equation (2) in the class of mappings from 2-divisible abelian groups to Banach spaces.

Given an integer $\lambda \neq 1$, Srisawat, Kitisin and Nakmahachalasint studied the solution of the alternative Jensen's functional equation of the form ${ }^{8}$

$$
\begin{aligned}
f\left(x y^{-1}\right)-2 f(x)+f(x y) & =0 \text { or } \\
f\left(x y^{-1}\right)-2 f(x)+\lambda f(x y) & =0
\end{aligned}
$$

when $f$ is a function from a group to a uniquely divisible abelian group, but the stability problem has not yet been investigated. This paper aims to prove the Hyers-Ulam stability of the alternative Jensen's functional equation (4) when $f$ is a mapping from a 2-divisible abelian group $(G, \cdot)$ to a Banach space $(E,\|\cdot\|)$. In other words, for every $\varepsilon \geqslant 0$, we show that there exist $\delta_{1}, \delta_{2} \geqslant 0$ such that if a mapping $f: G \rightarrow E$ satisfies the inequalities

$$
\begin{gathered}
\left\|f\left(x y^{-1}\right)-2 f(x)+f(x y)\right\| \leqslant \delta_{1} \quad \text { or } \\
\left\|f\left(x y^{-1}\right)-2 f(x)+\lambda f(x y)\right\| \leqslant \delta_{2}
\end{gathered}
$$

for all $x, y \in G$, then there exists a unique Jensen's mapping $J: G \rightarrow E$ with

$$
\|f(x)-J(x)\| \leqslant \varepsilon
$$

for all $x \in G$.

\section{AUXILIARY LEMMAS}

Let $(G, \cdot)$ be a group and $(E,\|\cdot\|)$ be a Banach space. Given an integer $\lambda$ and a function $f: G \rightarrow E$, for every pair $x, y \in G$ we define

$$
\mathscr{F}_{y}^{(\lambda)}(x):=\left\|f\left(x y^{-1}\right)-2 f(x)+\lambda f(x y)\right\| .
$$


For $\delta_{1}, \delta_{2} \geqslant 0$ and $\lambda \neq 1$, we write

$$
\begin{gathered}
\mathscr{S}_{y}^{(\lambda)}(x):=\left(\mathscr{F}_{y}^{(1)}(x) \leqslant \delta_{1} \text { or } \quad \mathscr{F}_{y}^{(\lambda)}(x) \leqslant \delta_{2}\right), \\
\mathscr{M}_{\delta_{1}, \delta_{2}}^{\lambda}:=\left(8+19|\lambda|+14 \lambda^{2}+3\left|\lambda^{3}\right|\right) \delta_{1} \\
+\left(61+88|\lambda|+31 \lambda^{2}+3\left|\lambda^{3}\right|\right) \delta_{2}
\end{gathered}
$$

and we denote the statement

$\mathscr{A}_{(G, E)}^{(\lambda)}:=\left\{f: G \rightarrow E \mid \mathscr{S}_{y}^{(\lambda)}(x)\right.$ for all $\left.x, y \in G\right\}$.

We first prove two lemmas concerning $\mathscr{S} f_{y}^{(\lambda)}(x)$.

Lemma 1 Let $f \in \mathscr{A}_{(G, E)}^{(\lambda)}$ and $x, y \in G$. Given $\alpha \geqslant 0$, if $\mathscr{F}_{y}^{(\lambda)}(x) \leqslant \delta_{2}$ and $\|f(x y)\| \leqslant \alpha$, then

$$
\mathscr{F}_{y}^{(1)}(x) \leqslant \delta_{2}+(1+|\lambda|) \alpha .
$$

Proof: Assume that $\mathscr{F}_{y}^{(\lambda)}(x) \leqslant \delta_{2}$ and $\|f(x y)\| \leqslant \alpha$. Thus

$$
\begin{aligned}
\left\|f\left(x y^{-1}\right)-2 f(x)\right\| & \leqslant \mathscr{F}_{y}^{(\lambda)}(x)+\|-\lambda f(x y)\| \\
& \leqslant \delta_{2}+|\lambda| \alpha .
\end{aligned}
$$

By $\|f(x y)\| \leqslant \alpha$ and (7), we obtain

$$
\begin{aligned}
\mathscr{F}_{y}^{(1)}(x) & \leqslant\left\|f\left(x y^{-1}\right)-2 f(x)\right\|+\|f(x y)\| \\
& \leqslant \delta_{2}+(1+|\lambda|) \alpha .
\end{aligned}
$$

Lemma 2 Let $f \in \mathscr{A}_{(G, E)}^{(\lambda)}$ and $x, y \in G$. If $\mathscr{F}_{y}^{(1)}(x)>$ $\delta_{1}$, then $\left\|f\left(x y^{-1}\right)-f(x y)\right\| \leqslant 2 \delta_{2}$.

Proof: Assume that $\mathscr{F}_{y}^{(1)}(x)>\delta_{1}$. The alternatives in $\mathscr{S} f_{y^{-1}}^{(\lambda)}(x)$ and $\mathscr{S} f_{y}^{(\lambda)}(x)$ give

$$
\mathscr{F}_{y^{-1}}^{(\lambda)}(x) \leqslant \delta_{2}, \quad \mathscr{F}_{y}^{(\lambda)}(x) \leqslant \delta_{2},
$$

respectively. Eliminating $f(x)$ from (8), we obtain

$$
\left\|(1-\lambda)\left(f\left(x y^{-1}\right)-f(x y)\right)\right\| \leqslant 2 \delta_{2} .
$$

Since $|1-\lambda| \geqslant 1$, we must have

$$
\left\|f\left(x y^{-1}\right)-f(x y)\right\| \leqslant 2 \delta_{2}
$$

as desired.

Next, we prove four lemmas concerning $\mathscr{S} f_{y^{2}}^{(\lambda)}(x)$.
Lemma 3 Let $f \in \mathscr{A}_{(G, E)}^{(\lambda)}$ and $x, y \in G$.

(i) If $\mathscr{F}_{y^{2}}^{(1)}(x)>\delta_{1}$ and $\mathscr{F}_{y}^{(1)}(x y)>\delta_{1}$, then $\mathscr{F}_{y^{2}}^{(1)}(x) \leqslant 6 \delta_{2}$.

(ii) If $\mathscr{F}_{y^{2}}^{(1)}(x)>\delta_{1}$ and $\mathscr{F}_{y}^{(1)}\left(x y^{-1}\right)>\delta_{1}$, then $\mathscr{F}_{y^{2}}^{(1)}(x) \leqslant 6 \delta_{2}$.

Proof: Case (i). Assume that $\mathscr{F}_{y^{2}}^{(1)}(x)>\delta_{1}$ and $\mathscr{F}_{y}^{(1)}(x y)>\delta_{1}$. By Lemma 2 , we obtain

$$
\begin{aligned}
\left\|f\left(x y^{-2}\right)-f\left(x y^{2}\right)\right\| & \leqslant 2 \delta_{2}, \\
\left\|f(x)-f\left(x y^{2}\right)\right\| & \leqslant 2 \delta_{2},
\end{aligned}
$$

respectively. From the above inequality, we obtain

$$
\left\|f\left(x y^{-2}\right)-2 f(x)+f\left(x y^{2}\right)\right\| \leqslant 6 \delta_{2} .
$$

Hence $\mathscr{F}_{y^{2}}^{(1)}(x) \leqslant 6 \delta_{2}$.

Case (ii). The proof is as in case (i) after replacing $y$ by $y^{-1}$.

Lemma 4 Let $f \in \mathscr{A}_{(G, E)}^{(\lambda)}$ and $x, y \in G$. If $\mathscr{F}_{y^{2}}^{(1)}(x)>$ $\delta_{1}$ and $\mathscr{F}_{y}^{(1)}(x) \leqslant \delta_{1}$, then

$$
\mathscr{F}_{y^{2}}^{(1)}(x) \leqslant 6 \max \left\{\delta_{1}, \delta_{2}\right\} .
$$

Proof: Assume that $\mathscr{F}_{y^{2}}^{(1)}(x)>\delta_{1}$ and $\mathscr{F}_{y}^{(1)}(x) \leqslant \delta_{1}$. We first consider the alternatives in $\mathscr{S} f_{y}^{(\lambda)}\left(x y^{-1}\right)$ and $\mathscr{S}_{y}^{(\lambda)}(x y)$ as follows. If $\mathscr{F}_{y}^{(1)}\left(x y^{-1}\right)>\delta_{1}$ or $\mathscr{F}_{y}^{(1)}(x y)>\delta_{1}$, then Lemma 3 gives $\mathscr{F}_{y^{2}}^{(1)}(x) \leqslant$ $6 \delta_{2}$. Then we assume that $\mathscr{F}_{y}^{(1)}\left(x y^{-1}\right) \leqslant \delta_{1}$ and $\mathscr{F}_{y}^{(1)}(x y) \leqslant \delta_{1}$. Thus

$\mathscr{F}_{y^{2}}^{(1)}(x) \leqslant \mathscr{F}_{y}^{(1)}\left(x y^{-1}\right)+2 \mathscr{F}_{y}^{(1)}(x)+\mathscr{F}_{y}^{(1)}(x y) \leqslant 6 \delta_{1}$.

Lemma 5 Let $f \in \mathscr{A}_{(G, E)}^{(-1)}$ and $x, y \in G$. If $\mathscr{F}_{y}^{(1)}(x)>$ $\delta_{1}$ and $\mathscr{F}_{y}^{(1)}(x y) \leqslant \delta_{1}$, then

$$
\left\|f\left(x y^{2}\right)\right\| \leqslant \max \left\{3 \delta_{1}+6 \delta_{2}, 5 \delta_{1}+4 \delta_{2}\right\} .
$$

Proof: Assume that $\mathscr{F}_{y}^{(1)}(x)>\delta_{1}$ and $\mathscr{F}_{y}^{(1)}(x y) \leqslant$ $\delta_{1}$. From $\mathscr{F}_{y}^{(1)}(x)>\delta_{1}$, Lemma 2 gives

$$
\left\|f\left(x y^{-1}\right)-f(x y)\right\| \leqslant 2 \delta_{2} .
$$

By $\mathscr{F}_{y}^{(1)}(x)>\delta_{1}$, the alternatives in $\mathscr{S} f_{y}^{(-1)}(x)$ and $\mathscr{S} f_{y^{-1}}^{(-1)}(x)$ give $\mathscr{F}_{y}^{(-1)}(x) \leqslant \delta_{2}$ and $\mathscr{F}_{y^{-1}}^{(-1)}(x) \leqslant \delta_{2}$, respectively. Hence

$$
\|2 f(x)\| \leqslant \frac{1}{2}\left(\mathscr{F}_{y}^{(-1)}(x)+\mathscr{F}_{y^{-1}}^{(-1)}(x)\right) \leqslant \delta_{2} .
$$


From (11) and $\mathscr{F}_{y}^{(1)}(x y) \leqslant \delta_{1}$ we obtain

$$
\left\|4 f(x y)-2 f\left(x y^{2}\right)\right\| \leqslant 2 \delta_{1}+\delta_{2} .
$$

Next, we will consider the following two possible cases in $\mathscr{S} f_{y}^{(-1)}\left(x y^{2}\right)$.

Case (i). Assume that $\mathscr{F}_{y}^{(-1)}\left(x y^{2}\right) \leqslant \delta_{2}$. Eliminating $f\left(x y^{2}\right)$ from (12) and $\mathscr{F}_{y}^{(-1)}\left(x y^{2}\right) \leqslant \delta_{2}$, we have

$$
\left\|3 f(x y)+f\left(x y^{3}\right)\right\| \leqslant 2 \delta_{1}+2 \delta_{2} .
$$

By (10) and (13), we obtain

$$
\left\|f\left(x y^{-1}\right)+2 f(x y)+f\left(x y^{3}\right)\right\| \leqslant 2 \delta_{1}+4 \delta_{2}
$$

and

$$
\left\|f\left(x y^{-1}\right)-4 f(x y)-f\left(x y^{3}\right)\right\| \leqslant 2 \delta_{1}+4 \delta_{2} .
$$

Consider $\mathscr{S} f_{y^{2}}^{(-1)}(x y)$ as follows. The alternative $\mathscr{F}_{y^{2}}^{(1)}(x y) \leqslant \delta_{1}$ and (14) give

$$
\|4 f(x y)\| \leqslant 3 \delta_{1}+4 \delta_{2},
$$

while the alternative $\mathscr{F}_{y^{2}}^{(-1)}(x y) \leqslant \delta_{2}$ and (15) give

$$
\|2 f(x y)\| \leqslant 2 \delta_{1}+5 \delta_{2} .
$$

By (12), (16) and (17), we obtain

$$
\left\|f\left(x y^{2}\right)\right\| \leqslant 3 \delta_{1}+6 \delta_{2} .
$$

Case (ii). Assume that $\mathscr{F}_{y}^{(1)}\left(x y^{2}\right) \leqslant \delta_{1}$. Eliminating $f\left(x y^{2}\right)$ from (12) and $\mathscr{F}_{y}^{(1)}\left(x y^{2}\right) \leqslant \delta_{1}$, we have

$$
\left\|3 f(x y)-f\left(x y^{3}\right)\right\| \leqslant 3 \delta_{1}+\delta_{2} .
$$

By (10) and (18), we obtain

$$
\left\|f\left(x y^{-1}\right)-4 f(x y)+f\left(x y^{3}\right)\right\| \leqslant 3 \delta_{1}+3 \delta_{2}
$$

and

$$
\left\|f\left(x y^{-1}\right)+2 f(x y)-f\left(x y^{3}\right)\right\| \leqslant 3 \delta_{1}+3 \delta_{2},
$$

follows. The alternative $\mathscr{F}_{y^{2}}^{(1)}(x y) \leqslant \delta_{1}$ and (19) give

$$
\|2 f(x y)\| \leqslant 4 \delta_{1}+3 \delta_{2},
$$

while the alternative $\mathscr{F}_{y^{2}}^{(-1)}(x y) \leqslant \delta_{2}$ and (20) give

$$
\|4 f(x y)\| \leqslant 3 \delta_{1}+4 \delta_{2} .
$$

By (12), (21) and (22), we obtain

$$
\left\|f\left(x y^{2}\right)\right\| \leqslant 5 \delta_{1}+4 \delta_{2} .
$$

From the two cases, we have (9) as desired.
Lemma 6 Let $f \in \mathscr{A}_{(G, E)}^{(\lambda)}$ and let $x, y \in G$. If $\mathscr{F}_{y^{2}}^{(1)}(x)>\delta_{1}$ and $\mathscr{F}_{y}^{(1)}(x)>\delta_{1}$, then

$$
\mathscr{F}_{y^{2}}^{(1)}(x) \leqslant \mathscr{M}_{\delta_{1}, \delta_{2}}^{\lambda} .
$$

Proof: From $\mathscr{F}_{y}^{(1)}(x)>\delta_{1}$, we obtain

$$
\left\|f\left(x y^{-1}\right)-f(x y)\right\| \leqslant 2 \delta_{2}
$$

by Lemma 2. We will consider the alternatives in $\mathscr{S} f_{y}^{(\lambda)}(x y)$ as follows. If $\mathscr{F}_{y}^{(1)}(x y)>\delta_{1}$, then Lemma 3 give $\mathscr{F}_{y^{2}}^{(1)}(x) \leqslant 6 \delta_{2}$ which satisfies (23). Thus we assume that $\mathscr{F}_{y}^{(1)}(x y) \leqslant \delta_{1}$. First, suppose that $\lambda=-1$. From $\mathscr{F}_{y}^{(1)}(x)>\delta_{1}$ and $\mathscr{F}_{y}^{(1)}(x y) \leqslant \delta_{1}$, by Lemma 5 , we obtain

$$
\left\|f\left(x y^{2}\right)\right\| \leqslant \max \left\{3 \delta_{1}+6 \delta_{2}, 5 \delta_{1}+4 \delta_{2}\right\} .
$$

Second, suppose that $\lambda \neq-1$. Since $\mathscr{F}_{y}^{(1)}(x)>\delta_{1}$, the alternatives in $\mathscr{S}_{y}^{(\lambda)}(x)$ gives $\mathscr{F}_{y}^{(\lambda)}(x) \leqslant \delta_{2}$. By (24) and $\mathscr{F}_{y}^{(\lambda)}(x) \leqslant \delta_{2}$, we obtain

$$
\|2 f(x)-(1+\lambda) f(x y)\| \leqslant 3 \delta_{2} .
$$

Eliminating $f(x)$ from (25) and $\mathscr{F}_{y}^{(1)}(x y) \leqslant \delta_{1}$, we obtain

$$
\left\|(3-\lambda) f(x y)-2 f\left(x y^{2}\right)\right\| \leqslant 2 \delta_{1}+3 \delta_{2} .
$$

Next, we will consider the following two possible cases in $\mathscr{S} f_{y}^{(\lambda)}\left(x y^{-1}\right)$.

Case (i). Assume that $\mathscr{F}_{y}^{(1)}\left(x y^{-1}\right)>\delta_{1}$. Since $\mathscr{F}_{y^{2}}^{(1)}(x)>\delta_{1}$ and $\mathscr{F}_{y}^{(1)}\left(x y^{-1}\right)>\delta_{1}$, Lemma 3 gives $\mathscr{F}_{y^{2}}^{(1)}(x) \leqslant 6 \delta_{2}$ which satisfies (23).

Case (ii). Assume that $\mathscr{F}_{y}^{(1)}\left(x y^{-1}\right) \leqslant \delta_{1}$. We eliminate $f\left(x y^{-1}\right)$ and $f(x)$ from (24), (25) and $\mathscr{F}_{y}^{(1)}\left(x y^{-1}\right) \leqslant \delta_{1}$ to obtain

$$
\left\|2 f\left(x y^{-2}\right)-(3-\lambda) f(x y)\right\| \leqslant 2 \delta_{1}+11 \delta_{2} .
$$

From $\mathscr{F}_{y^{2}}^{(1)}(x)>\delta_{1}$, the alternative $\mathscr{S} f_{y^{2}}^{(\lambda)}(x)$ gives $\mathscr{F}_{y^{2}}^{(\lambda)}(x) \leqslant \delta_{2}$. Then we eliminate $f(x)$ and $f\left(x y^{2}\right)$ from (25), (26) and $\mathscr{F}_{y^{2}}^{(\lambda)}(x) \leqslant \delta_{2}$ to obtain

$$
\begin{aligned}
\left\|2 f\left(x y^{-2}\right)-\left(2-\lambda+\lambda^{2}\right) f(x y)\right\| \\
\leqslant 2|\lambda| \delta_{1}+(8+3|\lambda|) \delta_{2} .
\end{aligned}
$$

From (27) and (28), we obtain

$\left\|\left(1-\lambda^{2}\right) f(x y)\right\| \leqslant(2+2|\lambda|) \delta_{1}+(19+3|\lambda|) \delta_{2} . \quad(29)$ 
Since $\left|1-\lambda^{2}\right| \geqslant 1$, (29) simplifies to

$$
\|f(x y)\| \leqslant(2+2|\lambda|) \delta_{1}+(19+3|\lambda|) \delta_{2} .
$$

From (26) and (30), we use $|\lambda-3| \leqslant|\lambda|+3$ to obtain $\left\|f\left(x y^{2}\right)\right\| \leqslant\left(8+11|\lambda|+3 \lambda^{2}\right) \delta_{1}+\left(60+28|\lambda|+3 \lambda^{2}\right) \delta_{2}$.

From the above two cases, we obtain (31). Hence by Lemma 1 we obtain (23) as desired.

\section{HYERS-ULAM STABILITY}

In this section, we will prove the Hyers-Ulam stability of the alternative Jensen's functional equation (4). The following lemma is crucial for the main theorem.

Lemma 7 Let $(G, \cdot)$ be a 2-divisible group. If $f \in$ $\mathscr{A}_{(G, E)}^{(\lambda)}$, then $\mathscr{F}_{y}^{(1)}(x) \leqslant \mathscr{M}_{\delta_{1}, \delta_{2}}^{\lambda}$ for all $x, y \in G$.

Proof: Let $f \in \mathscr{A}_{(G, E)}^{(\lambda)}$ and $x, y \in G$. Since $G$ is a 2-divisible group, there exists $z \in G$ such that $y=$ $z^{2}$. Considering the alternatives in $\mathscr{S} f_{z^{2}}^{(\lambda)}(x)$ and $\mathscr{S} f_{z}^{(\lambda)}(x)$, the proof is complete by Lemma 4 and Lemma 6.

It should be remarked that the 2-divisibility of the group $(G, \cdot)$ is important. In fact, Srisawat ${ }^{8}$, Kitisin and Nakmahachalasint proved that (4) is equivalent to (3) when the domain of $f$ is a 2divisible group. For $\lambda=-3$, (4) becomes

$$
\begin{aligned}
f\left(x y^{-1}\right)-2 f(x)+f(x y) & =0 \quad \text { or } \\
f\left(x y^{-1}\right)-2 f(x)-3 f(x y) & =0 .
\end{aligned}
$$

However, when the domain of $f$ is not a 2-divisible group, (32) does not need to be equivalent to (3) as illustrated by the following example.

Example 1 Given $a \in E \backslash\{0\}$. Let $f: \mathbb{Z} \rightarrow E$ be a mapping such that

$$
f(n)=(-1)^{n} a \text { for all } n \in \mathbb{Z} .
$$

We will first prove that $f$ satisfies (32). Given $n, m \in$ $\mathbb{Z}$. If $m$ is odd, then we see that $n-m$ and $n+m$ have the same parity whereas $n$ and $n+m$ have the opposite. Hence $f(n-m)-2 f(n)-3 f(n+m)=0$. Otherwise, if $m$ is even, then $n-m, n, n+m$ all have the same parity, i.e., $f(n-m)-2 f(n)+f(n+m)=0$. Next, we will show that $f$ does not satisfy (3). It should be noted that $f(0)-2 f(1)+f(2)=4 a$. From $a \neq 0$, we obtain $4 a \neq 0$. Thus $f$ satisfies (32) but $f$ does not satisfy (3).
Next, we will prove the Hyers-Ulam stability of the alternative Jensen's functional equation (4) by the so-called direct method. The stability results of Jensen's functional equation can be found, for instance, in Ref. 9.

Theorem 1 Let $(G, \cdot)$ be a 2-divisible group. If $f \in$ $\mathscr{A}_{(G, E)}^{(\lambda)}$, then there exists a unique Jensen's mapping $J: G \rightarrow E$ satisfying (3) with $J(0)=f(0)$ such that

$$
\|f(x)-J(x)\| \leqslant 2 \mathscr{M}_{\delta_{1}, \delta_{2}}^{\lambda} \quad \forall x \in G .
$$

Furthermore, the mapping $J$ is given by

$$
J(x)=f(0)+\lim _{n \rightarrow \infty} \frac{1}{2^{n}}\left(f\left(x^{2^{n}}\right)-f(0)\right) \quad \forall x \in G .
$$

Proof: Assume that $f \in \mathscr{A}_{(G, E)}^{(\lambda)}$. By Lemma 7, we obtain $\mathscr{F}_{y}^{(1)}(x) \leqslant \mathscr{M}_{\delta_{1}, \delta_{2}}^{\lambda}$ for all $x, y \in G$, i.e.,

$$
\left\|f\left(x y^{-1}\right)-2 f(x)+f(x y)\right\| \leqslant \mathscr{M}_{\delta_{1}, \delta_{2}}^{\lambda} .
$$

We define a function $\tilde{f}: G \rightarrow E$ by

$$
\tilde{f}(x)=f(x)-f(0) \text { for all } x \in G .
$$

It can be observed that $\tilde{f}(0)=0$. Then for each $x, y \in G$, we have

$$
\left\|\frac{1}{2}\left(\tilde{f}(x y)+\tilde{f}\left(x y^{-1}\right)\right)-\tilde{f}(x)\right\| \leqslant \frac{1}{2} \mathscr{M}_{\delta_{1}, \delta_{2}}^{\lambda} .
$$

Put $y=x$ in (33). Using $\tilde{f}(0)=0$ we obtain

$$
\left\|\frac{\tilde{f}\left(x^{2}\right)}{2}-\tilde{f}(x)\right\| \leqslant \frac{1}{2} \mathscr{M}_{\delta_{1}, \delta_{2}}^{\lambda} .
$$

For each positive integer $n$ and each $x \in G$, we apply (34) to obtain

$$
\begin{aligned}
\left\|\frac{\tilde{f}\left(x^{2^{n}}\right)}{2^{n}}-\tilde{f}(x)\right\| & =\left\|\sum_{i=1}^{n}\left(\frac{\tilde{f}\left(x^{2^{i}}\right)}{2^{i}}-\frac{\tilde{f}\left(x^{2^{i-1}}\right)}{2^{i-1}}\right)\right\| \\
& \leqslant\left(1-\frac{1}{2^{n}}\right) \mathscr{M}_{\delta_{1}, \delta_{2}}^{\lambda} .
\end{aligned}
$$

Consider the sequence $\left\{2^{-n} f\left(x^{2^{n}}\right)\right\}$. For all positive integers $m, n$ and every $x \in X$, we use (35) to obtain

$$
\begin{aligned}
\left\|\frac{\tilde{f}\left(x^{2^{n+m}}\right)}{2^{n+m}}-\frac{\tilde{f}\left(x^{2^{n}}\right)}{2^{n}}\right\| & =\frac{1}{2^{n}}\left\|\frac{\tilde{f}\left(x^{2^{n} \cdot 2^{m}}\right)}{2^{m}}-\tilde{f}\left(x^{2^{n}}\right)\right\| \\
& \leqslant \frac{1}{2^{n}}\left(1-\frac{1}{2^{m}}\right) \mathscr{M}_{\delta_{1}, \delta_{2}}^{\lambda} .
\end{aligned}
$$

Hence $\left\{2^{-n} f\left(x^{2^{n}}\right)\right\}$ is a Cauchy sequence. We can define a function $\tilde{J}: G \rightarrow E$ by

$$
\tilde{J}(x)=\lim _{n \rightarrow \infty} \frac{\tilde{f}\left(x^{2^{n}}\right)}{2^{n}} \quad \forall x \in G .
$$


Replacing $x$ by $x^{2^{n}}$ and $y$ by $y^{2^{n}}$ in (33), we obtain

$$
\left\|\frac{1}{2}\left(\tilde{f}\left(x^{2^{n}} y^{2^{n}}\right)+\tilde{f}\left(x^{2^{n}} y^{-2^{n}}\right)\right)-\tilde{f}\left(x^{2^{n}}\right)\right\| \leqslant \frac{1}{2} \mathscr{M}_{\delta_{1}, \delta_{2}}^{\lambda} .
$$

Next, multiplying (36) by $2^{-n}$ and taking $n \rightarrow \infty$, we obtain

$$
\tilde{J}(x y)+\tilde{J}\left(x y^{-1}\right)-2 \tilde{J}(x)=0 .
$$

From (35), as $n \rightarrow \infty$, we have

$$
\|\tilde{f}(x)-\tilde{J}(x)\| \leqslant \mathscr{M}_{\delta_{1}, \delta_{2}}^{\lambda} \quad \forall x \in G .
$$

To show the uniqueness of $\tilde{J}$, let $\mathscr{J}: G \rightarrow E$ satisfy $\mathscr{J}(0)=0$ and

$$
\|\tilde{f}(x)-\mathscr{J}(x)\| \leqslant \mathscr{M}_{\delta_{1}, \delta_{2}}^{\lambda} \quad \forall x \in G .
$$

For every positive integer $n$, we have

$$
\tilde{J}\left(x^{2^{n}}\right)=2^{n} \tilde{J}(x), \quad \mathscr{J}\left(x^{2^{n}}\right)=2^{n} \mathscr{J}(x) .
$$

Hence

$$
\begin{aligned}
& \|\mathscr{J}(x)-\tilde{J}(x)\| \\
& \quad=\left\|\frac{1}{2^{n}}\left(\tilde{J}\left(x^{2^{n}}\right)-\tilde{f}\left(x^{2^{n}}\right)\right)-\frac{1}{2^{n}}\left(\tilde{f}\left(x^{2^{n}}\right)-\mathscr{J}\left(x^{2^{n}}\right)\right)\right\| \\
& \quad \leqslant \frac{1}{2^{n}}\left\|\tilde{f}\left(x^{2^{n}}\right)-\tilde{J}\left(x^{2^{n}}\right)\right\|+\frac{1}{2^{n}}\left\|\tilde{f}\left(x^{2^{n}}\right)-\mathscr{J}\left(x^{2^{n}}\right)\right\| \\
& \quad \leqslant \frac{2}{2^{n}} \mathscr{M}_{\delta_{1}, \delta_{2}}^{\lambda} .
\end{aligned}
$$

As $n \rightarrow \infty$ in (37), we have $\mathscr{J}(x)=\tilde{J}(x)$ for all $x \in G$. By defining a function $J: G \rightarrow E$ by $J(x)=$ $\tilde{J}(x)+f(0)$ for all $x \in G$, the proof is complete.

\section{REFERENCES}

1. Kannappan PL, Kuczma M (1974) On a functional equation related to the Cauchy equation. Ann Polon Math 30, 49-55.

2. Ger R (1977) On an alternative functional equation. Aequat Math 15, 145-62.

3. Forti GL (1979) La soluzione generale dell'equazione funzionale $\{c f(x+y)-a f(x)-b f(y)-d\}\{f(x+y)-$ $f(x)-f(y)\}=0$. Matematiche 34, 219-42.

4. Nakmahachalasint $P$ (2012) An alternative Jensen's functional equation on semigroups. Sci Asia 38, 408-13.

5. Ng CT (1990) Jensen's functional equation on groups. Aequat Math 39, 85-99.

6. Parnami JC, Vasudeva HL (1992) On Jensen's functional equation. Aequat Math 43, 211-8.

7. Nakmahachalasint $P$ (2013) Stability of an alternative Jensen's functional equation. Sci Asia 39, 643-8.
8. Srisawat C, Kitisin N, Nakmahachalasint P (2015) On an alternative functional equation related to the Jensen's functional equation. East West J Math 17, 185-93.

9. Jung SM (1998) Hyers-Ulam-Rassias stability of Jensen's equation and its application. Proc Am Math Soc 126, 3137-43. 